\title{
Strain Characterization of Potato virus $S$ Isolates from Tasmania, Australia
}

Susan J. Lambert and Jason B. Scott, Tasmanian Institute of Agricultural Research (TIAR), University of Tasmania-Cradle Coast campus, Burnie, Tasmania, 7320, Australia; Sarah J. Pethybridge, Botanical Resources Australia-Agricultural Services Pty. Ltd., Ulverstone, Tasmania, 7315, Australia; and Frank S. Hay, TIAR, University of Tasmania-Cradle Coast campus

\begin{abstract}
Lambert, S. J., Scott, J. B., Pethybridge, S. J., and Hay, F. S. 2012. Strain characterization of Potato virus $S$ isolates from Tasmania, Australia. Plant Dis. 96:813-819.

Potato virus $S$ (PVS) is prevalent within potato (Solanum tuberosum) production worldwide. Traditionally, PVS has been split into two strains, Ordinary $\left(\mathrm{PVS}^{\mathrm{O}}\right)$ and Andean $\left(\mathrm{PVS}^{\mathrm{A}}\right)$, based on reaction in herbaceous indicator species such as Chenopodium quinoa. However, recent research has identified further strain designations, such as PVS ${ }^{\mathrm{O}-}$ CS (Ordinary and Chenopodium systemic). Forty-four isolates of PVS were collected from potato seed lines in different geographical regions within Tasmania, Australia. Isolates were initially characterized by reactions in C. quinoa. Nineteen isolates were characterized as $\mathrm{PVS}^{\mathrm{O}}$, based on the development of local lesions and serological detection in inoculated leaves only. Three isolates were identified as $\mathrm{PVS}^{\mathrm{A}}$-like, based on local lesion development in inoculated leaves, mild mottling or chlorotic spots on noninoculated leaves, and serological detection in both inoculated and noninoculated leaves. Thirteen isolates produced no symptoms, and were detected serologically in inoculated leaves

only (PVS ${ }^{\mathrm{O}}$-like). Four isolates produced no symptoms but were detected serologically in both inoculated and noninoculated leaves ( $\mathrm{PVS}^{\mathrm{A}}$-like). Five isolates produced symptoms in inoculated leaves only but were detected serologically in both inoculated and noninoculated leaves (also $\mathrm{PVS}^{\mathrm{A}}$-like). The ability of isolates to infect tomato has also been used as a criterion to assist in PVS strain differentiation. A subsample of isolates $(n=16)$ was unable to infect tomato 'Grosse Lisse'. Seventeen isolates representative of these groupings based on reactions in $C$. quinoa were also characterized by coat-protein sequencing. Phylogenetic comparisons suggested that all isolates were $\mathrm{PVS}^{\mathrm{O}}$ rather than $\mathrm{PVS}^{\mathrm{A}}$. Therefore, whereas some of these PVS isolates were systemic in $C$. quinoa, findings from this study suggest that they were not $\mathrm{PVS}^{\mathrm{A}}$, and that only $\mathrm{PVS}^{\mathrm{O}}$ and $\mathrm{PVS}^{\mathrm{O}-\mathrm{CS}}$ isolates are present in Tasmania. The implications of this finding for disease management are discussed.
\end{abstract}

Potato virus $S$ (PVS; family Betaflexiviridae; genus Carlavirus) is one of the most common potato (Solanum tuberosum L.) viruses found infecting production worldwide $(3,21)$. The majority of PVS infections of potato are symptomless but nonspecific symptoms have been described, including chlorotic mottling of the leaves and rugosity on the lower leaf surface (7). The virus is believed to be transmitted in a nonpersistent manner by a range of aphid species, including Myzus persicae, Rhopalosiphum padi, Aphis fabae, and A. nasturtii $(21,45)$. However, mechanical transmission and vegetative propagation are also considered important for virus spread $(14,23,24)$

Approximately one-quarter of the Australian potato crop is produced in Tasmania. The provision of virus-free planting material for this crop is vitally important for maintaining competitive yields. For example, in other countries, the presence of PVS and Potato virus $X$ has been reported to reduce potato yields by up to $20 \%$ (40). However, despite best practices and the implementation of a virus certification scheme in the 1930s, the presence of PVS (and other viruses) has been documented (25), raising concern within the Tasmanian industry regarding the lack of strain information for this virus, which may provide key insights into the biology of the pathogen and its management.

Traditionally, two strains of PVS have been recognized: the ordinary $\left(\mathrm{PVS}^{\mathrm{O}}\right)$ and Andean $\left(\mathrm{PVS}^{\mathrm{A}}\right)$ (22). Until recently, these strains were differentiated on the basis of their ability to infect the herbaceous indicator species Chenopodium quinoa in a systemic manner following mechanical inoculation. For example, $\mathrm{PVS}^{\mathrm{A}}$ strains are able to systemically infect $C$. quinoa, whereas $\mathrm{PVS}^{\mathrm{O}}$ strains are reported to induce only local lesions $(5,11,22)$. A further biological

Corresponding author: F. S. Hay, E-mail: Frank.Hay@utas.edu.au

Accepted for publication 9 December 2011.

http://dx.doi.org/10.1094/PDIS-07-11-0573

(C) 2012 The American Phytopathological Society factor used to differentiate the two PVS strains but with contrasting utility is the ability of an isolate to infect tomato (Solanum lycopersicum) $(1,6,11,20,39)$.

PVS $^{A}$ strains were originally described in South America $(18,21)$. However, isolates that infect $C$. quinoa in a systemic manner (i.e., $\mathrm{PVS}^{\mathrm{A}}$-like) have been subsequently described in other countries, including the Netherlands (36), the United States $(22,38)$, New Zealand $(11,12)$, and, in breeders' lines, within the United Kingdom (38) and Germany (7,39). Differences in the abilities of $\mathrm{PVS}^{\mathrm{O}}$ and $\mathrm{PVS}^{\mathrm{A}}$ to infect herbaceous indicator species have been attributed to several areas of the genome, including the $\mathrm{N}$ terminal regions of the 7-kDa protein, coat protein $(\mathrm{CP})$, and 11$\mathrm{kDa}$ protein encoded by the $3^{\prime}$ terminal region of the genome, together with shifts in the open reading frame patterns $(5,13,27,31,32)$. These changes have been reported to correspond to sections of the genome responsible for encoding differential virus movement and symptom development (31). Further studies by Matoušek et al. (32) used molecular probing to investigate sequence variability of PVS isolates from Europe, demonstrating that isolates systemically infecting $C$. quinoa were genetically closely related to European isolates of $\mathrm{PVS}^{\mathrm{O}}$ and distant from Andean $\mathrm{PVS}^{\mathrm{A}}$ isolates. This work termed such isolates $\mathrm{PVS}^{\mathrm{CS}}(\mathrm{CS}=$ Chenopodium systemic) to differentiate them from genetically distinct $\mathrm{PVS}^{\mathrm{A}}$ isolates which were also systemic in $C$. quinoa, and $\mathrm{PVS}^{\mathrm{O}}$ isolates which were genetically similar but not systemic in C. quinoa. Further molecular studies on PVS isolates were conducted by Cox and Jones (5), comparing 53 complete CP nucleotide sequences of PVS, 13 of which originated from Australia. This study suggested the presence of two clades associated with $\mathrm{PVS}^{\mathrm{O}}$ and $\mathrm{PVS}^{\mathrm{A}}$, with seven subclades of $\mathrm{PVS}^{\mathrm{O}}$ and three (two monotypic) subclades of $\mathrm{PVS}^{\mathrm{A}}$. Based on biological characterization of these isolates, Cox and Jones (5) suggested that the ability to infect $C$. quinoa and C. amaranticolor systemically should not be used as a definitive characteristic to identify $\mathrm{PVS}^{\mathrm{A}}$, and that isolates which were systemic in Chenopodium spp. but genetically related to $\mathrm{PVS}^{\mathrm{O}}$ and distant from PVS ${ }^{\mathrm{A}}$ should be designated PVS ${ }^{\mathrm{O}-\mathrm{CS}}$ (Ordinary-Chenopodium systemic). Moreover, Cox and Jones (5) sug- 
gested that the term PVS $^{\mathrm{A}}$ be confined to a genetically distinct clade which included isolates of PVS $^{\mathrm{A}}$ from South America that were systemic in C. quinoa. They further suggested that isolates of $\mathrm{PVS}^{\mathrm{A}}$ may exist which were unable to infect Chenopodium spp. systemically, and could be designated as $\mathrm{PVS}^{\mathrm{A}-\mathrm{CL}}$.

The objective of this study was to characterize isolates of PVS in Tasmania using a complementary approach, including both information on biological reaction after inoculation to two species, C. quinoa and tomato, and sequencing of the $\mathrm{CP}$ gene. This information is vital to the design of improved management strategies for PVS in Tasmanian seed potato production, because PVS ${ }^{\mathrm{A}}$ has been reportedly associated with more severe symptoms on potato leaves which may, in turn, imply a more significant effect on yield than the $\mathrm{PVS}^{\mathrm{O}}$ strains. Moreover, $\mathrm{PVS}^{\mathrm{A}}$ isolates are believed to be efficiently transmitted by aphids $(7,37,39,45)$, which would also have implications for the design of appropriate management plans.

\section{Materials and Methods}

Isolate collection. Twenty tubers of potato 'Russet Burbank' were randomly collected from each of a range of seed lines within Tasmania, Australia between 2003 and 2005. Tubers were stored at $4^{\circ} \mathrm{C}$ for 5 months, after which dormancy was broken by placing tubers in individual containers at room temperature for 4 days prior to planting. Tubers were planted in $200-\mathrm{mm}$ pots in commercial potting mix and maintained at $20^{\circ} \mathrm{C}$ in the greenhouse. One leaflet from each plant $(0.1 \mathrm{~g})$ was collected and tested in duplicate for the presence of PVS by double-antibody sandwich enzyme-linked immunosorbent assay (DAS-ELISA) using polyclonal antisera (Agdia Inc., Elkhart, IN), according to the manufacturer's protocol. Absorbance at $405 \mathrm{~nm}$ was recorded for each well using a Titertek photometer (Flow Laboratories, Helsinki, Finland). Samples were considered positive if absorbance was greater than the mean absorbance of the controls plus three times the standard deviation of the buffer only (42).

Mechanical inoculation. $C$. quinoa and $S$. lycopersicum 'Grosse Lisse' plants were grown in potting mix in a greenhouse and maintained at 15 to $20^{\circ} \mathrm{C}$. Five plants of $C$. quinoa were inoculated at the 8- to 10-leaf stage with each of the PVS isolates $(n=$ 44). Additionally, five tomato plants were inoculated at the five- to seven-true leaf stage with each of 16 isolates. Prior to inoculation, C. quinoa and tomato seedlings were maintained in darkness for 24 h. Leaf material from PVS-infected source plants $(1 \mathrm{~g})$ was macerated in $0.1 \mathrm{M}$ potassium phosphate buffer, $\mathrm{pH}$ 7.6. Inoculum for each isolate was rubbed liberally onto three marked leaves of each plant, following dusting with Celite. Excess Celite was removed with water post inoculation. Potassium phosphate buffer and Celite only were also applied to an equal number of noninoculated control plants. Plants were visually scored at 4-day intervals for 4 weeks for symptom development on inoculated and noninoculated leaves.

DAS-ELISA using the protocol described above was conducted to test for PVS infection of the three inoculated leaves and three upper (noninoculated) leaves from each plant at 24 days after inoculation (DAI) from both inoculated and noninoculated plants. Leaves were stored at $4{ }^{\circ} \mathrm{C}$ prior to testing and processed within 48 $\mathrm{h}$ of collection.

PVS CP gene sequencing and phylogenetic analysis. Total RNA was extracted from plant foliage $(100 \mathrm{mg})$ grown from tubers infected with 17 of the PVS isolates. Leaf material was frozen in liquid nitrogen and ground to a fine powder with a mortar and pestle. RNA was extracted using the RNeasy Plant Mini Kit (Qiagen, Valencia, CA) according to manufacturer's protocol. Final extracts were eluted into 30 to $50 \mu$ of RNase-free water and stored at $-80^{\circ} \mathrm{C}$.

RNA sequences of the CP gene of PVS were obtained from GenBank (http://www.ncbi.nlm.nih.gov/) (Table 1). Sequences were aligned with Clustal W (44) and manually corrected where necessary. Polymerase chain reaction (PCR) primers for the amplification of both PVS strains were designed, targeting conserved regions of the CP gene (PVS-forward, PVS-F 5' ATGCCGCCT AAACCAGATCC 3'; and PVS-reverse, PVS-R 5' TGATTG
CGCACAATCTCAGC 3'). The sense primer comprised part of the primer S2 used by Heldák (16). The predicted size of the amplicon from these primers was $863 \mathrm{bp}$. Primer specificity was confirmed by a BLAST search of GenBank for compatible sequences.

Reverse-transcription (RT)-PCR with the PVS-specific primers (PVS-F/PVS-R) was conducted on RNA extracted from all isolates (Table 2), using a OneStep RT-PCR kit (Qiagen) according to manufacturer's protocol and conditions adapted from Heldák (16). RT was undertaken using a preheat cycle at $50^{\circ} \mathrm{C}$ for $30 \mathrm{~min}$. This was followed by initial PCR denaturation for $15 \mathrm{~min}$ at $95^{\circ} \mathrm{C}$ and 30 cycles of $1 \mathrm{~min}$ of denaturation at $94^{\circ} \mathrm{C}, 1 \mathrm{~min}$ of annealing at $55^{\circ} \mathrm{C}$, and $1 \mathrm{~min}$ of primer extension at $72^{\circ} \mathrm{C}$. All amplifications were undertaken with a GeneAmp 2400 thermocycler (Perkin Elmer, Waltham, MA). Excess nucleotides were removed from the amplification products using the QIAQuick PCR PurificationKit (Qiagen) following the manufacturer's specifications, and resuspended in a final volume of $30 \mu \mathrm{l}$. Following clean-up, amplification products were separated in $1.5 \%$ agarose gel in $1 \times$ Tris-acetate-EDTA running buffer at $80 \mathrm{~V}$ for $80 \mathrm{~min}$, stained with ethidium bromide $(0.5 \mathrm{mg} / \mathrm{liter})$, and visualized under a UV light. DNA concentration was quantified through electrophoresis and comparison with a molecular mass standard (Bio-Rad Laboratories, Hercules, CA).

DNA products were sequenced using GenomeLab Dye Terminator Cycle Sequencing with Quick Start Kit (Beckman Coulter Inc., Brea, CA) chemistry, also following the manufacturer's standard protocol. All PCR products were sequenced in both the sense and antisense directions, using the primers PVS-F and PVS-R, respectively, with each reaction containing approximately 50 fmol DNA. Sequences were read using a CEQ8000 Genetic Analysis System (Beckman Coulter, Inc.). Sense and antisense sequences were aligned and compared using BioEdit v. 7.0.9.0 software (15), and a single consensus sequence was constructed for each isolate. To check for PCR amplification errors, duplicate RNA extracts were amplified, sequenced, and compared for homology. Obtained sequences were deposited with GenBank (Table 1).

Sequences of PVS isolates used in this study were combined with available sequences of the PVS CP obtained from GenBank (Table 1). One isolate of Potato rough dwarf virus was included as an out-group for phylogenetic analyses. All sequences were aligned using Clustal W v. 1.4 (44). Alignment was manually corrected using BioEdit software, where necessary, cropped to a region present within all sequences, and coded to remove phylogenetic bias associated with sites of multibase indels introduced within the alignment.

Phylogenetic analysis of this alignment was conducted using both maximum parsimony and Bayesian inference methodologies. Maximum parsimony analysis was conducted with PAUP* v. $4.0 \mathrm{~b} 10$ (43). A heuristic search was used with 10 random sequence additions and tree bisection-reconnection branch swapping. A maximum of 20,000 trees was stored and ACCTRAN optimization was used. Indels were treated as a fifth character state. In all, 1,000 bootstrap pseudo-replicates were constructed and analyzed using the same methodology to assess the statistical strength of the observed phylogenetic tree topology (9). Bootstrap values greater than or equal to $70 \%$ were considered significant (17). Bayesian inference analysis was conducted in MRBAYES v. 3.1 (36), using a Metropolis-coupled Markov chain Monte Carlo methodology. Indels were coded as present $(=1)$ or absent $(=0)$ and the data set was divided into DNA and coded partitions. The optimal evolutionary model for analyzing the DNA partition was selected using MrModelTest v. 2 (35). The coded partition was analyzed using the standard discrete evolutionary model implemented in MRBAYES. Both partitions were analyzed together. Two concurrent analyses of four chains (one cold and three heated) were each run for $1,000,000$ generations, to ensure that analyses were not trapped at local optima (26). Both analyses were initiated with random starting trees and sampled every 100 generations. Log-likelihood scores of samples were plotted against generation to estimate the time point at which stationarity was reached for each analysis and, thus, 
determine the number of samples to be burned in prior to final analysis. A majority rule consensus tree was constructed following burn-in for both analyses. Clades in this tree with posterior probabilities equal to or greater than 0.95 were deemed significant (26). Resultant phylogenetic trees were visualized using TreeGraph v. 2.0.45-197b (41).

\section{Results}

Symptom development following inoculation to $C$. quinoa. All 44 PVS isolates were successfully inoculated into $C$. quinoa with high inoculation efficiency (Table 2). Nineteen isolates were characterized as $\mathrm{PVS}^{\mathrm{O}}$ based on local lesions on inoculated leaves of $C$. quinoa and the absence of systemic symptoms (Table 2). For these isolates, serological testing detected PVS in inoculated leaves only (Table 2). Symptoms produced on inoculated leaves were chlorotic local lesions and chlorotic mottling.

A further three isolates were putatively identified as $\mathrm{PVS}^{\mathrm{A}}$ based on the development of local lesions on inoculated leaves and mild chlorotic mottling or chlorotic spots on noninoculated leaves (Table 2). Serological testing confirmed the presence of PVS in both inoculated and noninoculated leaves (Table 2). Systemic symptoms induced by isolates identified putatively as $\mathrm{PVS}^{\mathrm{A}}$

Table 1. Potato virus $S$ (PVS) coat protein sequences obtained from GenBank for comparative purposes

\begin{tabular}{|c|c|c|c|}
\hline Isolate code (strain designation) & GenBank accession & Origin & Reference \\
\hline PVS3-5 (ordinary) & AB364945 & Aleppo, Syria & 4 \\
\hline PVS6-2 (unclassified) & AB364946 & Hama, Syria & 4 \\
\hline BoonO (ordinary) & AF493950 & Unknown & 2 \\
\hline Andean (Andean) & AF493951 & Unknown & 2 \\
\hline Leona (unclassified) & AJ863509 & Germany & 32 \\
\hline Vltava (unclassified) & AJ863510 & Czech Republic & Unpublished \\
\hline Hangzhou (unclassified) & AJ889246 & Hangzhou, China & Unpublished \\
\hline PVSHZ1220 (unclassified) & AY512653 & China & Unpublished \\
\hline PVSHZ00P1 (unclassified) & AY687337 & Hangzhou, China & Unpublished \\
\hline Andean (Andean) & D00461 & Peru & 28 \\
\hline Hebei (unclassified) & DQ315387 & Zhangjiakou, China & Unpublished \\
\hline PVS - O (ordinary) & DQ786653 & India & Unpublished \\
\hline Ar-43 (unclassified) & EF397748 & Iran & Unpublished \\
\hline Az-21 (unclassified) & EF397749 & Iran & Unpublished \\
\hline Es-81 (unclassified) & EF397750 & Iran & Unpublished \\
\hline Ha-107 (unclassified) & EF397751 & Iran & Unpublished \\
\hline Kh-55 (unclassified) & EF397752 & Iran & Unpublished \\
\hline Andean (India) (Andean) & FJ643622 & India & Unpublished \\
\hline WaDef-US (unclassified) & FJ813512 & Washington State, United States & 27 \\
\hline Id4106-US (unclassified) & FJ813513 & Washington State, United States & 27 \\
\hline IdDef-US (unclassified) & FJ813514 & Washington State, United States & 27 \\
\hline 136-PVS (unclassified) & GU144322 & Scotland & 34 \\
\hline 137-PVS (unclassified) & GU144323 & Scotland & 34 \\
\hline 138-PVS (unclassified) & GU144324 & Scotland & 34 \\
\hline 139-PVS (unclassified) & GU144325 & Scotland & 34 \\
\hline 143-PVS (unclassified) & GU144326 & Scotland & 34 \\
\hline 144-PVS (unclassified) & GU144327 & Scotland & 34 \\
\hline Sam-13-PVS (unclassified) & GU144328 & Scotland & 34 \\
\hline Sam-23-PVS (unclassified) & GU144329 & Scotland & 34 \\
\hline Sam-24-PVS (unclassified) & GU144330 & Scotland & 34 \\
\hline QLDKip (ordinary) & GU233453 & Queensland, Australia & 5 \\
\hline QLD1092 (unclassified) & GU233454 & Gatton, Queensland & 5 \\
\hline SK (unclassified) & GU233455 & Western Australia & 48 \\
\hline Indian (unclassified) & GU256061 & Himachal Pradesh, India & Unpublished \\
\hline TAS05-09 (ordinary) & GU319942 & Riana, Tasmania & 5 \\
\hline WADel1 (ordinary) & GU319943 & Kwinana, Western Australia & 5 \\
\hline WADel2 (unclassified) & GU319944 & Bornholme, Western Australia & 5 \\
\hline WAFL (unclassified) & GU319945 & Lake Sadie, Western Australia & 5 \\
\hline WAKip1 (ordinary) & GU319946 & Western Australia & 5 \\
\hline WAKip2 (unclassified) & GU319947 & Western Australia & 5 \\
\hline WAMd (unclassified) & GU319948 & Young's Siding, Western Australia & 5 \\
\hline WAPC (ordinary) & GU319949 & Western Australia & 5 \\
\hline TASPF (ordinary) & GU319950 & Tasmania & 5 \\
\hline Dutch (unclassified) & GU319951 & Netherlands & 8 \\
\hline Exodus (unclassified) & GU319952 & Netherlands & 8 \\
\hline Code 24 (ordinary) & GU319953 & Netherlands or Germany & 8,13 \\
\hline TAS1 (ordinary) & GU319954 & Tasmania & 5 \\
\hline O Indian (unclassified) & GU369814 & India & Unpublished \\
\hline Orion (ordinary) & S45593 & United Kingdom & 13 \\
\hline S-SE (unclassified) & U74375 & South Korea & Unpublished \\
\hline S-RB (unclassified) & U74376 & South Korea & Unpublished \\
\hline VLCP1N (ordinary) & Y15609 & Czech Republic & 31 \\
\hline VLCP2N (ordinary) & Y15610 & Czech Republic & 31 \\
\hline KACP1N (ordinary) & Y15611 & Czech Republic & 31 \\
\hline KACP2N (ordinary) & Y15612 & Czech Republic & 31 \\
\hline ASCP12 (ordinary) & Y15613 & Czech Republic & 31 \\
\hline $\mathrm{KBCP} 2$ (ordinary) & Y15614 & Czech Republic & 31 \\
\hline ASCP9 (ordinary) & Y15615 & Czech Republic & 31 \\
\hline VLCP2 (ordinary) & Y15616 & Czech Republic & 31 \\
\hline Kobra (ordinary) & Y15625 & Czech Republic & 31 \\
\hline Potato rough dwarf virus-Arg (outgroup) & EU020009 & Argentina & 29 \\
\hline
\end{tabular}


included faint mottling, chlorotic lesions on noninoculated leaves, and chlorosis of leaf tips. Symptoms developed on upper leaves from 21 DAI.

Of the remaining 25 isolates, 13 isolates were asymptomatic on both inoculated and noninoculated leaves; however, serological testing detected PVS in inoculated but not noninoculated leaves. On this basis, isolates were classified as $\mathrm{PVS}^{\mathrm{O}}$-like (Table 2). A further nine isolates were detected serologically in both inoculated and noninoculated leaves but lacked typical symptoms of $\mathrm{PVS}^{\mathrm{A}}$, and were classed as $\mathrm{PVS}^{\mathrm{A}}$-like. Of these, four isolates produced no symptoms on $C$. quinoa and five isolates produced symptoms on inoculated leaves only (Table 2). PVS was not detected in any of the noninoculated control plants.

Symptom development following inoculation to tomato. No symptoms were observed in tomato plants inoculated with 16 PVS isolates or on noninoculated control plants. This included two isolates (TAS03-NW40.5 and TAS03-NE59.3) putatively identified as $\mathrm{PVS}^{\mathrm{A}}$ based on systemic infection of $C$. quinoa (Table 2). Moreover, serological testing also failed to detect PVS in any of the noninoculated control plants.

PVS CP gene sequencing and phylogenetic analysis. Following alignment, cropping and coding, and multisequence alignment, $805 \mathrm{bp}$ remained for analysis. Within this alignment, 397 sites were homologous across all isolates. Of the 408 variable sites, 235 were parsimony informative, and 235 uninformative. Across all PVS isolates, sequence homology varied between 0.79 and 1.00 . Within Tasmanian PVS isolates sequenced in this study, sequence homology varied between 0.95 and 1.00 .

Maximum parsimony analysis resulted in 1,359 equally parsimonious trees, 947 steps in length (Fig. 1). Retention and consis-

Table 2. Isolate code, origin, year of collection, and infection of Chenopodium quinoa of Potato virus $S$ (PVS) isolates obtained from individual seed potato lines in Tasmania, Australia

\begin{tabular}{|c|c|c|c|c|c|c|c|}
\hline \multirow[b]{2}{*}{ Isolate ${ }^{c}$} & \multirow[b]{2}{*}{ Region of Tasmania } & \multirow[b]{2}{*}{$\begin{array}{c}\text { Year of } \\
\text { collection }\end{array}$} & \multicolumn{2}{|c|}{ Symptoms in C. quinoa ${ }^{a}$} & \multicolumn{2}{|c|}{ Serological detection $^{\mathbf{b}}$} & \multirow[b]{2}{*}{$\begin{array}{l}\text { GenBank } \\
\text { accession }\end{array}$} \\
\hline & & & $\begin{array}{l}\text { Inoculated } \\
\text { leaves }\end{array}$ & $\begin{array}{l}\text { Noninoculated } \\
\text { (systemic) leaves }\end{array}$ & $\begin{array}{l}\text { Inoculated } \\
\text { leaves }\end{array}$ & $\begin{array}{c}\text { Noninoculated } \\
\text { leaves }\end{array}$ & \\
\hline \multicolumn{8}{|l|}{$\mathrm{PVS}^{\mathrm{O}}$} \\
\hline TAS05-NW4.5 & Sheffield & 2005 & CLL & None & $1.0(0.93)$ & 0 & \\
\hline TAS03-NW6.4 & South Riana & 2003 & $\ldots$ & $\ldots$ & $1.0(0.73)$ & 0 & JN244086 \\
\hline TAS03-NW13.2 & Wilmot & 2003 & $\ldots$ & $\ldots$ & $1.0(1.00)$ & 0 & $\ldots$ \\
\hline TAS03-NW16.5 & Riana & 2003 & $\ldots$ & $\ldots$ & $1.0(1.00)$ & 0 & JN244097 \\
\hline TAS03-NW17.4 & South Riana & 2003 & $\ldots$ & $\ldots$ & $1.0(0.67)$ & 0 & $\ldots$ \\
\hline TAS05-NE22.4 & Scottsdale & 2005 & $\ldots$ & $\ldots$ & $1.0(1.00)$ & 0 & $\ldots$ \\
\hline TAS03-NW23.1 & Tunnack & 2003 & $\ldots$ & $\ldots$ & $1.0(0.93)$ & 0 & JN244099 \\
\hline TAS05-NW24.3 & Sheffield & 2005 & $\ldots$ & $\ldots$ & $1.0(0.93)$ & 0 & $\ldots$ \\
\hline TAS03-NW24.4 & Riana & 2003 & $\ldots$ & $\ldots$ & $1.0(1.00)$ & 0 & JN244094 \\
\hline TAS03-NW25.4 & Riana & 2003 & $\ldots$ & $\ldots$ & $0.8(0.80)$ & 0 & JN244095 \\
\hline TAS03-NW35.3 & Preston & 2003 & $\ldots$ & $\ldots$ & $0.8(0.67)$ & 0 & $\ldots$ \\
\hline TAS03-NW40.4 & Lower Barrington & 2003 & $\ldots$ & $\ldots$ & $1.0(0.67)$ & 0 & 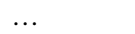 \\
\hline TAS03-NE49.2 & Scottsdale & 2003 & $\ldots$ & $\ldots$ & $1.0(1.00)$ & 0 & JN244098 \\
\hline TAS05-NW50.2 & Riana & 2005 & $\ldots$ & $\ldots$ & $1.0(0.93)$ & 0 & $\ldots$ \\
\hline TAS03-NE56.1 & Riana & 2005 & $\ldots$ & $\ldots$ & $1.0(0.93)$ & 0 & $\ldots$ \\
\hline TAS05-NE56.1 & Scottsdale & 2005 & $\ldots$ & $\ldots$ & $1.0(0.93)$ & 0 & $\ldots$ \\
\hline TAS05-NE57.5 & Tullendeena & 2005 & $\ldots$ & $\ldots$ & $1.0(1.00)$ & 0 & $\ldots$ \\
\hline TAS03-NE60.1 & St. Helens & 2003 & $\ldots$ & $\ldots$ & $1.0(1.00)$ & 0 & $\ldots$ \\
\hline TAS05-NE60.1 & St. Helens & 2005 & $\ldots$ & $\ldots$ & $1.0(1.00)$ & 0 & JN244088 \\
\hline \multicolumn{8}{|l|}{$\mathrm{PVS}^{\mathrm{O}}$-like } \\
\hline TAS03-NW7.5 & Upper Natone & 2003 & None & None & $0.2(0.13)$ & 0 & JN244087 \\
\hline TAS05-NW11.2 & Sheffield & 2005 & $\ldots$ & $\ldots$ & $1.0(1.00)$ & 0 & $\ldots$ \\
\hline TAS05-NW17.2 & Ridgley & 2005 & $\ldots$ & $\ldots$ & $1.0(1.00)$ & 0 & $\ldots$ \\
\hline TAS05-NE25.1 & West Scottsdale & 2005 & $\ldots$ & $\ldots$ & $1.0(1.00)$ & 0 & $\ldots$ \\
\hline TAS05-NW28.3 & Upper Natone & 2005 & $\ldots$ & $\ldots$ & $1.0(0.93)$ & 0 & $\ldots$ \\
\hline TAS05-NW31.5 & Riana & 2005 & $\ldots$ & $\ldots$ & $1.0(1.00)$ & 0 & JN244090 \\
\hline TAS03-NW35.2 & Preston & 2003 & $\ldots$ & $\ldots$ & $0.8(0.47)$ & 0 & JN244092 \\
\hline TAS05-NW41.2 & Yolla & 2005 & $\ldots$ & $\ldots$ & $1.0(1.00)$ & 0 & $\ldots$ \\
\hline TAS05-S48.1 & WoodStock & & $\ldots$ & $\ldots$ & $1.0(1.00)$ & 0 & $\ldots$ \\
\hline TAS03-NE53.2 & Scottsdale & 2003 & $\ldots$ & $\ldots$ & $1.0(0.87)$ & 0 & $\ldots$ \\
\hline TAS05-NE57.4 & Tullendeena & 2005 & $\ldots$ & $\ldots$ & $1.0(1.00)$ & 0 & $\ldots$ \\
\hline TAS05-NE58.2 & Winnaleah & 2005 & $\ldots$ & $\ldots$ & $1.0(0.93)$ & 0 & $\ldots$ \\
\hline TAS03-NE58.5 & Winnaleah & 2003 & $\ldots$ & $\ldots$ & $0.8(0.60)$ & 0 & JN244091 \\
\hline \multicolumn{8}{|l|}{$\mathrm{PVS}^{\mathrm{A}}$-like } \\
\hline TAS03-NW40.5 & Lower Barrington & 2003 & CLL & CLL and mottling & $1.0(0.67)$ & $1.0(0.40)$ & JN244084 \\
\hline TAS03-NE50.3 & Branxholm & 2003 & $\ldots$ & $\ldots$ & $1.0(1.00)$ & $0.8(0.58)$ & \\
\hline TAS03-NE59.3 & Winnaleah & 2003 & $\ldots$ & $\ldots$ & $1.0(1.00)$ & $0.4(0.13)$ & JN244093 \\
\hline TAS03-NW4.3 & Sheffield & 2003 & None & None & $1.0(1.00)$ & $0.4(0.13)$ & JN244085 \\
\hline TAS03-NW17.2 & South Riana & 2003 & None & $\ldots$ & $1.0(0.87)$ & $0.4(0.13)$ & $\ldots$ \\
\hline TAS03-NW22.1 & Sheffield & 2003 & CLL & $\ldots$ & $1.0(0.87)$ & $0.2(0.07$ & JN244089 \\
\hline TAS03-NW28.1 & Wilmot & 2003 & CLL and mottling & $\ldots$ & $1.0(0.73)$ & $0.2(0.20)$ & JN244096 \\
\hline TAS03-NW31.5 & Calder & 2003 & None & $\ldots$ & $1.0(0.87)$ & $0.4(0.13)$ & $\ldots$ \\
\hline TAS03-NW41.3 & Yolla & 2003 & CLL & $\ldots$ & $0.8(0.80)$ & $0.2(0.20)$ & $\ldots$ \\
\hline TAS03-NE52.1 & Scottsdale & 2003 & Chlorosis & $\ldots$ & $1.0(1.00)$ & $0.6(0.40)$ & $\mathrm{JN} 244100$ \\
\hline TAS03-NE56.3 & Riana & 2003 & CLL & $\ldots$ & $1.0(1.00)$ & $1.0(0.67)$ & $\ldots$ \\
\hline TAS03-NE59.2 & Winnaleah & 2003 & None & $\ldots$ & $1.0(0.53)$ & $0.4(0.13)$ & $\ldots$ \\
\hline
\end{tabular}

${ }^{a}$ Observed symptoms for each PVS group match those given for the first listed isolate of that group, unless otherwise indicated. CLL $=$ chlorotic local lesions.

${ }^{\mathrm{b}}$ Inoculation efficiency as quantified by double-antibody sandwich enzyme-linked immunosorbent assay. Incidence of PVS-infected plants $(n=5)$. Values presented parenthetically are mean proportions of inoculated or noninoculated leaves per plant with PVS ( $n=3$ per plant).

${ }^{\mathrm{c}}$ Isolates were grouped as PVS-Ordinary (PVS ${ }^{\mathrm{O}}$; symptoms and serological detection in inoculated leaves only), PVS ${ }^{\mathrm{O}}$-like (asymptomatic but detected serologically in inoculated leaves only), or PVS-Andean $\left(\mathrm{PVS}^{\mathrm{A}}\right)$-like (symptoms or serological detection in both inoculated and noninoculated leaves; variable between isolates). 


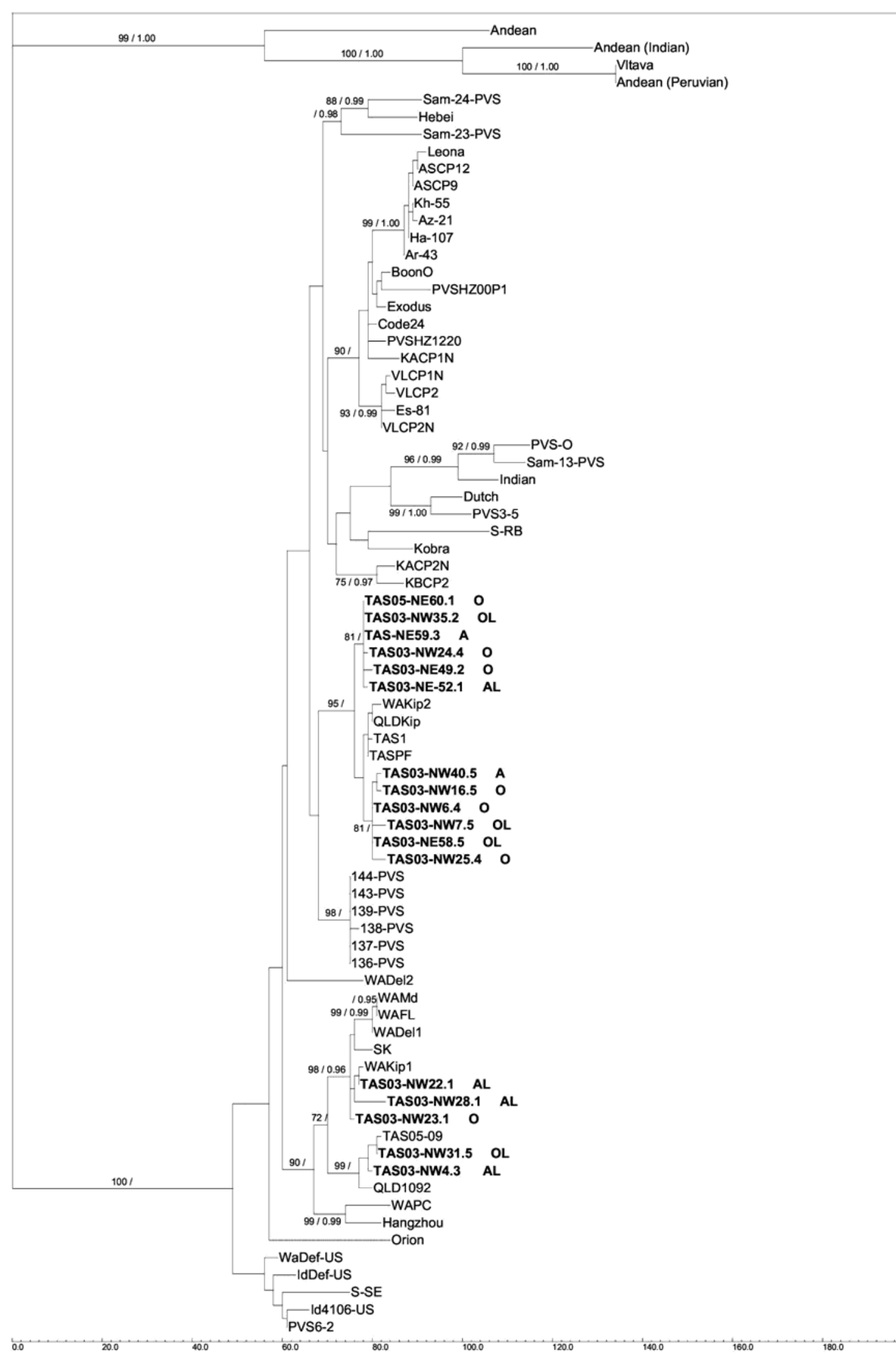


tency indices were 0.773 and 0.599 , respectively. Superimposing bootstrap (BS) values from maximum parsimony analysis and posterior probabilities (PP) from analysis conducted by Bayesian inference indicated that all Andean strain isolates of PVS from GenBank were located in a single well-supported clade $(\mathrm{BS}=$ 99\%; PP = 1.00), distinct from all other PVS isolates (Fig. 1). Maximum parsimony analysis located all $\mathrm{PVS}^{\mathrm{O}}$ isolates within a single clade $(\mathrm{BS}=100 \%$ ), although this was not supported by Bayesian inference. Within the PVS ${ }^{\mathrm{O}}$ clade, isolates were not consistently grouped, with both BS and PP support, into subclades. Tasmanian PVS isolates sequenced in this study grouped into the PVS $^{\mathrm{O}}$ clade and were separated into four subclades, two of which were supported by high BS values and one by PP. These clades also contained some but not all isolates of Australian origin obtained from GenBank. No international isolates grouped with Tasmanian isolates. The subclades contained isolates sourced from a range of different geographic regions and contained isolates of both $\mathrm{PVS}^{\mathrm{O}}$ and $\mathrm{PVS}^{\mathrm{O}-\mathrm{CS}}$ (Table 2; Fig. 1).

\section{Discussion}

To the best of our knowledge, this study constitutes the first report of PVS isolates in Australia which are capable of systemically infecting $C$. quinoa. Sequencing of the $\mathrm{CP}$ demonstrated that all Tasmanian isolates were distinct from the clade containing all sequences of international $\mathrm{PVS}^{\mathrm{A}}$ isolates. Moreover, Tasmanian isolates of PVS in this study which infected C. quinoa systemically grouped with other Tasmanian isolates which did not and, therefore, can be designated PVS ${ }^{\mathrm{O}-\mathrm{CS}}$ according to the nomenclature of Cox and Jones (5) rather than being considered PVS ${ }^{\mathrm{A}}$. The inability of any of the isolates in this study to infect tomato provides further support of this recommendation. However, evidence for the use of this characteristic in PVS isolate differentiation has been highly confused. For example, Slack (39) reported that, in general, PVS isolates from North America and Europe which induced a local lesion response in $C$. quinoa were not able to infect tomato $(1,6)$, whereas Horváth $(20)$ reported that European isolates were able to asymptomatically infect only some Lycopersicon spp. (syn. S. lycopersicum). Other studies have used the inability of PVS to infect tomato following mechanical inoculation as a means of separating PVS and Potato virus $M(1,47)$. Moreover, Fletcher (11) reported an isolate of PVS from New Zealand which was systemic in $C$. quinoa and aphid transmissible and could infect and produce symptoms in tomato, whereas another isolate which was not systemic in $C$. quinoa was unable to infect. In our study, we were unable to infect Grosse Lisse tomato with 16 Tasmanian isolates of PVS, regardless of their ability to systemically infect $C$. quinoa. This was not the cultivar used in previous PVS isolate characterization studies (e.g., Matousek et al. [33] used 'Nevski') and, hence, there may be potential for variation between the ability of various isolates to infect systemically with cultivar.

Phylogenetic analyses further separated Tasmanian $\mathrm{PVS}^{\mathrm{O}}$ isolates into four subclades, well supported by bootstrapping but not by Bayesian inference in all cases. These clades also contained some but not all sequences of isolates of Australian origin obtained from GenBank. No international isolates grouped with Tasmanian isolates. Subclades were not related to the region in which seed lines were grown, with various regions around Tasmania represented within each subclade. Similarly, subclades did not appear to be related to whether isolates were classified as $\mathrm{PVS}^{\mathrm{O}}$ or $\mathrm{PVS}^{\mathrm{O}-\mathrm{CS}}$ based on reaction in $C$. quinoa, with both being present within individual subclades. This finding further supports those of Cox and Jones (5) that also suggested, despite high variability in the Nterminal region between isolates, that $\mathrm{CP}$ sequences of PVS are not indicative of the ability of an isolate to systemically infect $C$. quinoa.

An additional key finding from this study was that some isolates of PVS are able to infect C. quinoa systemically, and be detected serologically in noninoculated leaves, without producing symptoms. Moreover, some isolates produced no symptoms on $C$. quinoa but could be detected serologically in inoculated leaves only.
Expression of local lesions in strains of PVS not systemic in $C$. quinoa may be affected by a range of factors, including the $C$.quinoa seed source (30) and incubation conditions following inoculation (19), and, hence, there may be potential for variation between both PVS isolates and tomato cultivars in the ability to establish systemic infections. Therefore, it is likely that such factors would also affect systemic symptom development. However, this suggests that characterization of PVS strains based on symptom expression only following mechanical inoculation to $C$. quinoa and possibly other herbaceous indicator species may be unreliable and should include other means of virus detection, such as serology and sequencing of multiple genes. Recent work by Matoušek et al. (30) has provided strong evidence that the triple-gene block of PVS is an important determinant of host range or systemic movement of this virus, which would be a suitable candidate for further studies.

The presence of only PVS ${ }^{\mathrm{O}}$ and $\mathrm{PVS}^{\mathrm{O}-\mathrm{CS}}$ isolates in Tasmania, which may be less efficiently aphid transmitted than PVS ${ }^{\mathrm{A}}$ isolates (46), is also consistent with the limited spread of this virus observed in Tasmanian potato fields (25) compared with temporal spread rates in other studies $(10,11,14,23)$. Moreover, the significant spatiotemporal association of PVS-infected plants described in Tasmanian potato fields provides further evidence for limited within-field spread, including the presence of isolates capable of being transmitted with high efficiency by aphids (25). Together, this work supports findings suggesting that the prevalence and incidence of PVS in Tasmanian potato fields may be substantially reduced by the maintenance of an effective seed certification scheme and use of elite virus-free planting stock.

Further work to clarify the importance of biological differences conferred by PVS strains, including effects on yield and quality, and determination of the genetic basis of aphid transmission would be beneficial for the implementation of disease management strategies within potato production.

\section{Acknowledgments}

This work was facilitated through Horticulture Australia Ltd., and supported financially from grower's levy, Simplot Australia Pty. Ltd., McCain Foods (Australia) Pty. Ltd., Harvest Moon Forth Farm Produce Pty. Ltd., with matching funding from the Australian Commonwealth Government. Additional financial support was obtained from the University of Tasmania's Institutional Research Grants Scheme. This study formed part of a Doctor of Philosophy degree for S. J. Lambert.

\section{Literature Cited}

1. Bagnall, R. H., Larson, R. H., and Walker J. C., 1956. Potato viruses $M, S$ and $X$ in relation to interveinal mosaic of the Irish Cobbler variety. Wis. Univ. Agric. Exp. Stn. Res. Bull. 198.

2. Boonham, N., Walsh, K., Smith, P., Madagan, K., Graham, I., and Barker, I. 2003. Detection of potato viruses using microarray technology: towards a generic method for plant viral disease diagnosis. J. Virol. Methods 108:181187.

3. Brunt, A. A., and Loebenstein, G. 2001. The main viruses infecting potato crops. Pages 65-133 in: Viruses and virus-like diseases of potato and production of seed potatoes. G. Loebenstein, P. H. Berger, A. A. Brunt, and R. H. Lawson, eds. Kluwer, Dordrecht, The Netherlands.

4. Chikh Ali, M., Maoka, T., and Natsuaki, K. 2008. The occurrence of potato viruses in Syria and the molecular detection and characterization of Syrian Potato virus $S$ isolates. Pot. Res. 51:151-161.

5. Cox, B. A., and Jones, R .A. C. 2010. Genetic variability in the coat protein gene of Potato virus $S$ isolates and distinguishing its biologically distinct strains. Arch. Virol. 155:1163-1169.

6. De Bokx, J. A. 1970. Reactions of various plant species to inoculation with Potato Virus S. Neth. J. Plant Pathol. 76:70-78.

7. Dolby, C. A., and Jones, R. A. C. 1987. Occurrence of the Andean strain of Potato virus $S$ in imported potato material and its effects on potato cultivars. Plant Pathol. 36:381-388.

8. Dolby, C. A., and Jones, R. A. C. 1988. The relationship between the Andean strain of Potato virus $S$ and Pepino latent virus. Ann. Appl. Biol 112:231-234.

9. Felsenstein, J. 1985. Confidence-limits on phylogenies—an approach using the bootstrap. Evolution 39:783-91.

10. Fletcher, J. D. 1984. Levels of virus incidence in pathogen tested and group 1 seed potatoes in New Zealand. Aust. Plant Pathol. 13:33-35.

11. Fletcher, J. D. 1996. Potato virus $S^{\mathrm{A}}$ - characterisation of an isolate from New Zealand. N.Z. J. Crop Hortic. Sci. 24:335-339.

12. Fletcher, J. D., Lethwaite, S. L., Boddington, H. J., Nott, H. M., and Wood, R. J. 1996. Virus disease surveys of ware potato crops, Franklin County, 
North Island, New Zealand. N. Z. J. Crop Hortic. Sci. 24:7-12.

13. Foster, G. D., and Mills, P. R. 1992. The 3'-nucleotide sequence of an ordinary strain of Potato virus $S$. Virus Genes 6:213-220.

14. Franc, G. D., and Banttari, E. E. 1996. Translocation and mechanical spread of a Minnesota isolate of Potato virus $S$ in potatoes. Am. Pot. J. 73:123-133.

15. Hall, T. A. 1999. BioEdit: a user-friendly biological sequence alignment editor and analysis program for Windows 95/98/NT. Nucleic Acids Symp. Ser. 41:95-98.

16. Heldák, J. 2001. Detection of potato virus S by RT-PCR in potato regenerants derived from in vitro heat-treated shoot tips. Acta Fytotechnica et Zootechnica, Vol. 4. Pages 275-277 in: Proc. Int. Sci. Conf. Occasion of the 55th Anniversary of the Slovak Agricultural University in Nitra.

17. Hillis, D. M., and Bull, J. J. 1993. An empirical test of bootstrapping as a method for assessing confidence in phylogenetic analysis. Syst. Biol. 42:182-192.

18. Hinostroza-Orihuela, A. M. 1973. Some properties of Potato virus $S$ isolated from Peruvian potato varieties. Pot. Res. 16:244-250.

19. Hiruki, C. 1975. Factors affecting bioassay of Potato virus $S$ in Chenopodium quinoa. Phytopathology 65:1288-1292.

20. Horváth, J. 1972. Symptomless Lycopersicon host plants for Potato virus S. Am. J. Pot. Res. 49:339-341.

21. Jones, R. A. C. 1981. The ecology of viruses infecting wild and cultivated potatoes in the Andean regions of South America. Pages 89-107 in: Pests Pathogens and Vegetation. J. M. Thresh, ed. Pitman, London.

22. Jones, R. A. C., Fribourg, C. E., and Slack, S. A. 1982. Potato virus and virus-like diseases. In: Plant Virus Slide Series, Set No. 2. O. W. Barnett and S. A. Tollin, eds. Clemson University, South Carolina.

23. Kamenikova, L. 1978. The spread of Potato virus S. Ochr. Rostl. (Wyd. 4) 14:101-106.

24. Khalil, E. M., and Shalla, T. A. 1982. Detection and spread of Potato virus S. Plant Dis. 66:368-371.

25. Lambert, S. J., Hay, F. S., Pethybridge, S. J., and Wilson, C. R. 2007. Spatiotemporal spread of Potato virus $S$ and Potato virus $X$ in seed potato in Tasmania, Australia. Online. Plant Health Progress. Online publication. doi:10.1094/PHP-2007-0726-07-RS.

26. Leache, A. D., and Reeder, T. W. 2002. Molecular systematics of the Eastern Fence Lizard (Sceloporus undulatus): a comparison of parsimony, likelihood, and Bayesian approaches. Syst. Biol. 51:44-68.

27. Lin, Y. H., Druffel, K. L., Whitworth, J., Pavek, M. J., and Pappu, H. R. 2009. Molecular characterization of two potato virus S isolates from lateblight-resistant genotypes of potato (Solanum tuberosum). Arch. Virol. 154:1861-1863.

28. Mackenzie, D. J., Tremaine, J. H., and Stacesmith, R. 1989. Organization and interviral homologies of the 3'-terminal portion of potato virus S RNA. J. Gen. Virol. 70:1053-1063.

29. Massa, G. A., Segretin, M. E., Colavita, M., Riero, M. F., BravoAlmonacid, F., and Feingold, S. 2006. Biological and sequence data suggest that potato rough dwarf virus (PRDV) and potato virus $\mathrm{P}$ (PVP) are strains of the same species. Arch. Virol. 151:1243-1247.

30. Matoušek, J., Schubert, J., and Dědič, P. 2009. Complementation analysis of triple gene block of Potato virus $S$ (PVS) revealed its capability to support systemic infection and aphid transmissibility of recombinant Potato virus $X$ Virus Res. 146:81-88.

31. Matoušek, J., Schubert, J., Dědič, P., and Ptáček, J. 2000. A broad variability of Potato virus $S$ (PVS) revealed by analysis of virus sequences amplified by reverse transcriptase-polymerase chain reaction. Can. J. Plant Pathol. 22:29-37.

32. Matoušek, J., Schubert, J., Ptáček, J., Kozlová, P., and Dědič, P. 2005. Complete sequence and molecular probing of Potato virus $S$ (PVS) genome. Acta Virol. 49:195-205.

33. Matsumoto, T., and Hiruki, C. 1980. Chenopodium quinoa strains suitable for quantitative assay of potato virus S. Pot. Res. 23:49-55.

34. Mortensen, R. J., Shen, X. Y, Reid, A., and Mulholland, V. 2010. Characterization of viruses infecting potato plants from a single location in Shetland, an isolated Scottish archipelago. J. Phytopathol. 158:633-640.

35. Nylander, J. A. A. 2004. MrModeltest v. 2 V. v2: Program distributed by the author. Evolutionary Biology Centre, Uppsala University.

36. Ronquist, F., and Huelsenbeck, J.P. 2003. MrBayes 3: Bayesian phylogenetic inference under mixed models. Bioinformatics 19:1572-4.

37. Rose, D. G. 1983. Some properties of an unusual isolate of potato virus $\mathrm{S}$ Pot. Res. 26:49-62.

38. Slack, S. 1981. Identification of an unusual strain of potato virus $\mathrm{S}$ in North America. (Abstr.) Phytopathology 71:255.

39. Slack, S. A. 1983. Identification of an isolate of the Andean strain of potato virus $\mathrm{S}$ in North America. Plant Dis. 67:786-789.

40. Stevenson, W. R., Loria, R., Franc, G. D., and Weingartner, D. P., eds. 2001. Compendium of Potato Diseases. American Phytopathological Society, St. Paul, MN.

41. Stover, B., and Muller, K. 2010. TreeGraph 2: combining and visualizing evidence from different phylogenetic analyses. BMC Bioinf. 11:7.

42. Sutula, C. L., Gillet, J. M., Morrissey, S. M., and Ramsdel, D. C. 1986 Interpreting ELISA data and establishing the positive-negative threshold. Plant Dis. 70:722-726.

43. Swofford, D. L. 2003. PAUP*. Phylogenetic Analysis Using Parsimony (*and Other Methods), Version 4. Sinauer Associates, Sunderland, MA

44. Thompson, J. D., Higgins, D. G., and Gibson, T. J. 1994. CLUSTAL W: Improving the sensitivity of progressive multiple sequence alignment through sequence weighting, position-specific gap penalties and weight matrix choice. Nucleic Acids Res. 22:4673-80.

45. Wardrop, E. A., Gray, A. B., Singh, R. P., and Peterson, J. F. 1989. Aphid transmission of Potato virus $S$. Am. Pot. J. 66:449-459.

46. Weidemann, H.-L., and Koenig, R. 1990. Differentiation of isolates of potato virus $\mathrm{S}$ which infect Chenopodium quinoa systemically by means of quantitative cDNA hybridization tests. Z. Pflanzenkrankh. Pflanzenschutz 97:323-327.

47. Wetter, C. 1972. Potato virus M. Descriptions of Plant Viruses. No. 60 Commonwealth Mycological Institute/Association of Applied Biologists, Kew, Surrey, England.

48. Wilson, C. R., and Jones, R. A. C., 1993. Resistance to potato leafroll virus infection and accumulation in potato cultivars, and the effects of previous infection with other viruses on expression of resistance. Aust. J. Agric. Res 44:1891-904 